\title{
IRAS Identification of Complete Samples of Cool Carbon Stars: Results for a Region in Carina
}

\author{
Francisco J. Fuenmayor \\ Departamento de Física, Facultad de Ciencias, Universidad de Los \\ Andes, Mérida 5101, Venezuela \\ Jurgen Stock \\ Centro de Investigaciones de Astronomia (CIDA), Mérida 5101, \\ Venezuela
}

\begin{abstract}
Results for a near-infrared objective-prism survey for faint cool carbon stars in a Carina region centred at $l=290^{\circ}, b=0^{\circ}$ are presented. A total of 37 identified carbon stars in area of about 16 square degrees were found, of which 4 are new discoveries. Accurate positions, IRAS identifications, infrared fluxes and colours, and associations of the stars in the sample are given.
\end{abstract}

\section{Introduction}

Interest in the study of the space distribution of Asymptotic Giant Branch (AGB) stars has grown primarily because it is believed they are relevant to our understanding of the late stages of stellar evolution, -and the formation and evolution of stellar systems. AGB stars are evolved objects of low effective temperature and high luminosity, which represent a stellar population in an evolutionary stage between main sequence and white dwarf stars. During the AGB phase a star can lose a significant amound of its initial mass, surrounding itself with a circumstellar envelope (CSE) which sometimes may be dense enough as to preclude its observation in visible light (Jura \& Kleinmann 1989).

$\mathrm{M}, \mathrm{C}$, and S stars together represent the largest fraction of known AGB stars, and they are found mainly in the galactic disk. However, a marked difference exists between galactic distribution of $M$ stars and the cooler $C$ stars, in the sense that the number density ratio $C / M$ is not constant throughout the galactic disk ( $\mathrm{C} / \mathrm{M}$ increase towards the outer regions of the Galaxy), contrary to what is to be expected from an evolutionary relationship between these stars (Blanco 1965, 1989; Fuenmayor 1981).

Usually, low-dispersion surveys made in the near-infrared neglect to identify the late M-type stars, their number being overwhelming on the same plates where $\mathrm{C}$ and $\mathrm{S}$ stars are found. Therefore, most of the determinations of the space density ratios between $M$ and $C$ stars given in the literature have not been directly determined. In this paper we present the results of a deep search of carbon stars in a Carina region at $l=290^{\circ}$, as part of a project to survey 
selected regions along the galactic plane to determine accurate positions, spectral types and IRAS identifications for AGB stars.

\section{Observations}

The region under study covers an area of 16 square degrees centred at RA $=11 \mathrm{~h} 10 \mathrm{~m}$ and dec $=-61^{\circ}(1950)$, with galactic coordinates $l=290^{\circ}, b=0^{\circ}$. Two CTIO plates, listed in Table I, were used for this search. Identification of carbon stars were made under a binocular Zeiss microscope. Carbon stars are identified on near-infrared emulsions by the presence of the CN strong bandheads at $\lambda \lambda 7945,8125,8320 \AA$. No attempt was made to classify the carbon stars thus found into subclasses, but most of these stars correspond to N-type, which are among the reddest and coolest carbon stars. XY coordinates of the identified stars, together with standard stars, were made using a Zeiss PSK machine at CIDA by measuring the position of the telluric $A$ band on the unwidened spectra. Plate coordinates were converted into equatorial coordinates using a concentric plate-projection method devised by Stock (1981). The PPM catalogue was used as the source for standard positions. Accurate positions were thus obtained with a mean error of 0.1 arcsec. Near-infrared I magnitudes were estimated directly from the density of the spectral image. Carbon stars were identified almost up to the plate limiting magnitude $I=13$. Cross identifications were performed with both IRAS Point Source Catalog and the IRAS Low Resolution Spectra Catalog as well. Software implemented at the SUMA Computer Center at ULA and CIDA was used to search catalogues.

Table I. CTIO Plates used in this Survey

\begin{tabular}{llcll}
\hline Plate No. & Emulsion & Filter & Exposure & Prism \\
& & & & \\
\hline CTIO 21368 & IV-N+ & RG-68 & 60 min & $4^{\circ}$ \\
CTIO 21378 & IV-N & RG-68 & 15 & $4^{\circ}$ \\
\hline
\end{tabular}

\section{A Complete Sample of Carbon Stars in a Region in Carina}

In Table II a list of a complete sample of 37 cool carbon stars identified in the region under study is presented. Columns 2 and 3 denote the equatorial coordinates at the equinox 1950. The estimated I magnitude is given in column 4. IRAS $12 \mu \mathrm{m}$ magnitude with no colour correction is given in column 5. In columns 6 and 7 IRAS colour indices [12-25] and [25 - 60] are shown. Columns 8 and 9 show the results for cross identifications with the IRAS Point Source Catalogue (1989) and Stephenson's General Catalogue of Galactic Cool Carbon Stars (CCCS) (1989), respectively. Stars marked 23 through 26 are new identifications. 
Table II. A list of cool carbon stars in a region in Carina

\begin{tabular}{|c|c|c|c|c|c|c|c|c|c|c|c|c|}
\hline No. & & R.7 & & 9501 & & ec. & I & [12] [ & $12-25]$ & $25-6$ & IRAS SOURCE & cccs \\
\hline 1 & 10 & 50 & 27.49 & -62 & 35 & 28.4 & 12.1 & 4.22 & 1.31 & 3.02 & $10504-6235$ & 2884 \\
\hline 2 & 10 & 50 & 27.84 & -59 & 57 & 10.6 & 12.9 & 3.01 & 0.86 & 4.54 & $10504-5956$ & 2883 \\
\hline 3 & 10 & 50 & 40.49 & -63 & 0 & 2.4 & 12.6 & 4.54 & 0.90 & 3.55 & $10506-6259$ & 2887 \\
\hline 4 & 10 & 50 & 53.07 & -62 & 4 & 18.7 & 10.7 & & & & & 2888 \\
\hline 5 & 10 & 51 & 31.60 & -60 & 41 & 28.9 & 11.9 & 3.95 & 2.82 & 4.04 & $10515-6041$ & 2889 \\
\hline 6 & 10 & 51 & 55.60 & -61 & 11 & 31.6 & 10.3 & 3.53 & 2.15 & 4.39 & $10519-6111$ & 2894 \\
\hline 7 & 10 & 54 & 34.45 & -60 & 47 & 9.7 & 10.7 & & & & & 2903 \\
\hline 8 & 10 & 55 & 58.18 & -61 & 38 & 27.1 & 12.1 & & & & & 2913 \\
\hline 9 & 10 & 56 & 32.45 & -62 & 19 & 4.1 & 10.3 & 3.66 & 0.50 & 3.52 & $10565-6219$ & 2915 \\
\hline 10 & 10 & 56 & 47.47 & -62 & 23 & 5.1 & 8.7 & 1.13 & 0.21 & 0.76 & $10567-6222$ & 2916 \\
\hline 11 & 10 & 57 & 2.90 & -60 & 16 & 3.7 & 12.1 & & & & & 2917 \\
\hline 12 & 10 & 59 & 13.93 & -62 & 42 & 49.0 & 10.7 & 4.20 & 0.97 & 3.60 & $10592-6242$ & 2929 \\
\hline 13 & 10 & 59 & 43.14 & -61 & 21 & 4.7 & 12.9 & & & & & 2933 \\
\hline 14 & 11 & 0 & 6.20 & -61 & 37 & 17.0 & 12.2 & 4.07 & 2.56 & 3.88 & $11001-6137$ & 2935 \\
\hline 15 & 11 & 0 & 39.47 & -59 & 13 & 54.8 & 12.1 & & & & & 2939 \\
\hline 16 & 11 & 0 & 49.75 & -60 & 39 & 38.1 & 12.1 & & & & & 2940 \\
\hline 17 & 11 & 0 & 57.61 & -61 & 17 & 28.8 & 11.8 & 0.85 & 1.01 & 2.68 & $11009-6117$ & 2941 \\
\hline 18 & 11 & 2 & 33.59 & -59 & 53 & 54.3 & 10.7 & & & & & 2948 \\
\hline 19 & 11 & 3 & 29.78 & -61 & 5 & 3.0 & 9.9 & & & & & 2950 \\
\hline 20 & 11 & 3 & 51.60 & -60 & 44 & 23.4 & 12.9 & & & & & 2952 \\
\hline 21 & 11 & 4 & 33.88 & -61 & 15 & 21.2 & 12.1 & & & & & 2955 \\
\hline 22 & 11 & 4 & 42.48 & -58 & 18 & 43.1 & 9.2 & 3.32 & 0.38 & 3.79 & $11047-5818$ & 2956 \\
\hline 23 & 11 & 6 & 4.72 & -61 & 19 & 29.4 & 12.1 & & & & & \\
\hline 24 & 11 & 8 & 46.46 & -58 & 38 & 26.2 & 12.9 & & & & & \\
\hline 25 & 11 & 14 & 8.14 & -58 & 35 & 32.7 & 11.4 & & & & & \\
\hline 26 & 11 & 14 & 23.57 & -58 & 45 & 46.8 & 12.9 & & & & & \\
\hline 27 & 11 & 18 & 19.93 & -59 & 14 & 47.4 & 12.9 & & & & & 2997 \\
\hline 28 & 11 & 18 & 26.62 & -60 & 27 & 5.3 & 12.3 & & & & & 2999 \\
\hline 29 & 11 & 19 & 4.77 & -60 & 1 & 34.1 & 9.1 & 3.05 & 0.54 & 2.97 & $11190-6001$ & 3002 \\
\hline 30 & 11 & 19 & 50.58 & -59 & 22 & 41.2 & 11.4 & & & & & 3003 \\
\hline 31 & 11 & 23 & 58.03 & -59 & 12 & 2.7 & 11.2 & 4.45 & 0.82 & 4.28 & $11239-5911$ & 3017 \\
\hline 32 & 11 & 24 & 34.28 & -60 & 52 & 51.6 & 10.4 & 3.09 & 0.67 & 3.60 & $11245-6052$ & 3019 \\
\hline 33 & 11 & 25 & 29.67 & -59 & 2 & 35.9 & 11.8 & 4.66 & 1.30 & 3.69 & $11255-5902$ & 3024 \\
\hline 34 & 11 & 26 & 1.86 & -60 & 37 & 32.1 & 12.3 & 3.90 & 0.85 & 4.42 & $13260-6037$ & 3028 \\
\hline 35 & 11 & 26 & 41.97 & -59 & 27 & 18.7 & 11.4 & 4.33 & 0.70 & 4.23 & $11267-5927$ & 3032 \\
\hline 36 & 11 & 27 & 57.10 & -62 & 2 & 22.4 & 12.1 & & & & & 3039 \\
\hline 37 & 11 & 28 & 2.94 & -62 & 4 & 17.8 & 12.9 & 3.63 & 0.82 & 5.22 & $11280-6203$ & 3040 \\
\hline
\end{tabular}




\section{References}

Blanco V. M., 1965, in Stars and Stellar Systems, Vol 5, W. A. Blaaw \& M. Schmidt, eds, (Univ. Chicago Press, Chicago), p241

Blanco V. M., 1989, Rev. Mex. Astron. Ast., 19, 25

Fuenmayor F. J., 1981, Rev. Mex. Astron. Ast., 6, 83

IRAS Science Team, 1989, IRAS Point Source Catalog

Jura M. \& Kleinmann S. G., 1989, ApJ, 341, 359

Stock J., 1981, Rev. Mex. Astron. Ast., 6, 115 